\title{
Metastatic myxofibrosarcoma of the spermatic cord responding to mesna, adriamycin, ifosfamide, and dacarbazine combination therapy
}

\author{
Hideaki Ito $\cdot$ Nobuyuki Oyama $\cdot$ Yoshiji Miwa $\cdot$ \\ Hironobu Akino $\cdot$ Yoshiaki Imamura • \\ Osamu Yokoyama
}

Received: 27 October 2011/Accepted: 25 December 2011/Published online: 18 January 2012

(C) The Japan Society of Clinical Oncology 2012

\begin{abstract}
Myxofibrosarcoma is common in the extremities of elderly people and is characterized by a high frequency of local recurrence. We report herein a case of a 72-year-old male who presented with a 5-year history of right scrotal swelling and a month history of painful swelling in the scrotal area. Computed tomography scan revealed a scrotal tumor $5 \mathrm{~cm}$ in size and a tumor in the rectum. He subsequently underwent right high orchiectomy and rectal dissection with colostomy. The scrotal tumor was histopathologically diagnosed as a myxofibrosarcoma; the rectal tumor was a leiomyosarcoma. The patient underwent tumor resection for recurrence and left inguinal metastasis, but soon developed local recurrences and lymph node metastasis of the left iliac area. Chemotherapy with mesna, adriamycin, ifosfamide, and dacarbazine (MAID) resulted in partial remission. We report the rare case of myxofibrosarcoma in the spermatic cord for which chemotherapy with MAID was an effective treatment.
\end{abstract}

Keywords Spermatic cord · Myxofibrosarcoma · MAID

\section{Introduction}

Myxofibrosarcoma (MFS) is a fibroblast-derived sarcoma with myxoid areas in association with varying proportions

H. Ito $(\bowtie) \cdot$ N. Oyama · Y. Miwa · H. Akino · O. Yokoyama Department of Urology, Faculty of Medical Science, University of Fukui, Fukui, Japan

e-mail: uroito@u-fukui.ac.jp

Y. Imamura

The Division of Tumor Pathology, Department of Pathological Sciences, Faculty of Medical Science, University of Fukui, Fukui, Japan of cellular areas with a pleomorphic storiform pattern. It grows commonly in subcutaneous areas of the extremities of elderly people [1]. Surgery is the most common form of therapy; only a limited number of papers deal with chemotherapy, because of the small number of cases. The paucity of cases and the variety of tumor characteristics make it very difficult to analyze treatment modalities and outcome studies in a large series. We report herein a case of metastatic MFS in which chemotherapy with MAID resulted in partial remission (PR).

\section{Case report}

A 72-year-old man presented with a 5-year history of right inguinal to scrotal swelling and a month history of progressive painful swelling of the lesion. Examination revealed a hard swelling in the right inguinal to scrotum, which was separated from the right testis. The mass was smooth, hard, mobile and painful on pressure, not fluctuant. The inguinal lymph node was not palpable. There were no systemic symptoms. A contrast-enhanced CT scan revealed a well defined, heterogeneously enhanced, soft tissue mass $5 \mathrm{~cm}$ in size in a right inguinal to scrotal lesion (Fig. 1a). Screening for metastases revealed a mass $2 \mathrm{~cm}$ in diameter in the patient's rectum (Fig. 1b). Swollen lymph nodes were not detected.

The patient underwent a right high orchiectomy and rectal dissection with colostomy. Rapid intraoperative pathological diagnosis during the orchiectomy revealed no malignant tumor in the surgical margin. The excised specimen was $50 \times 45 \times 45 \mathrm{~mm}$, a yellowish collagenous mass intermingled with myxoid material, which was completely separated from the testis. Microscopically, the tumor was circumscribed. Myxoid areas in association with 

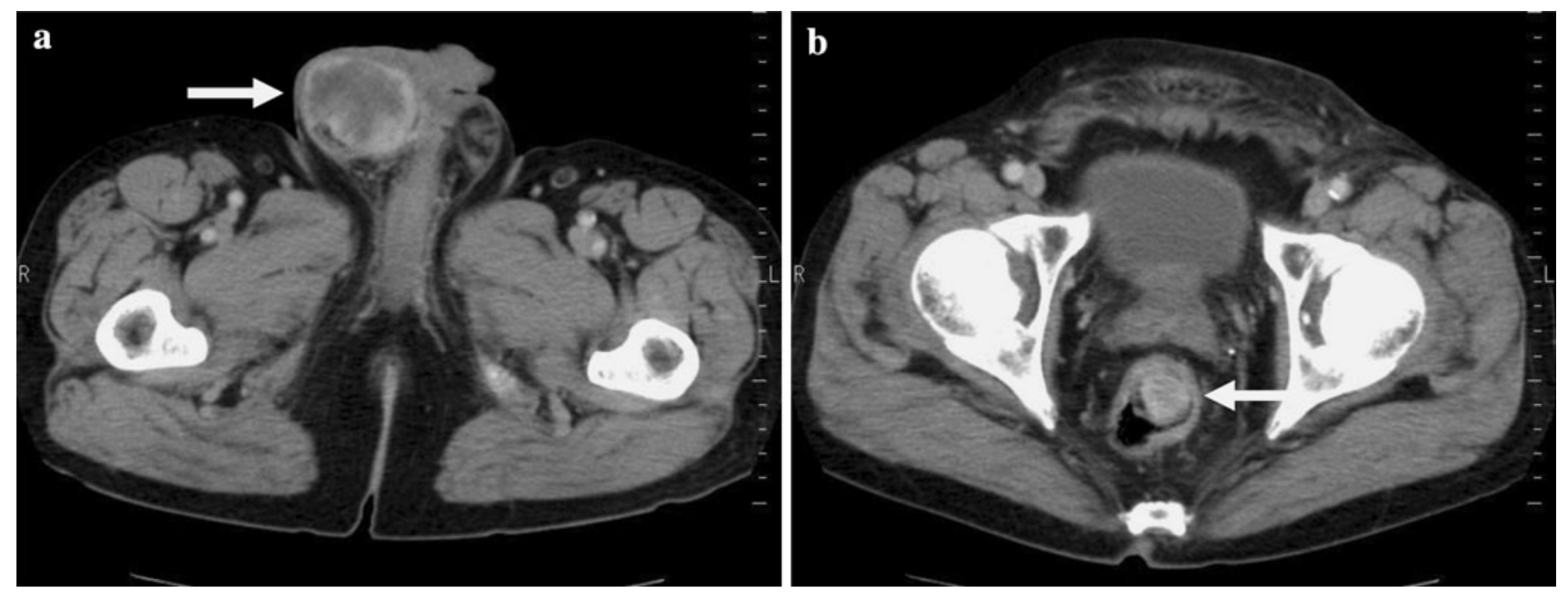

Fig. 1 Computed tomography (CT) scan of the patient. A contrast-enhanced CT scan revealed a well-defined soft tissue mass (arrow) in the right scrotal area (a). CT scan also revealed a mass (arrow) $2 \mathrm{~cm}$ in diameter in the patient's rectum (b)

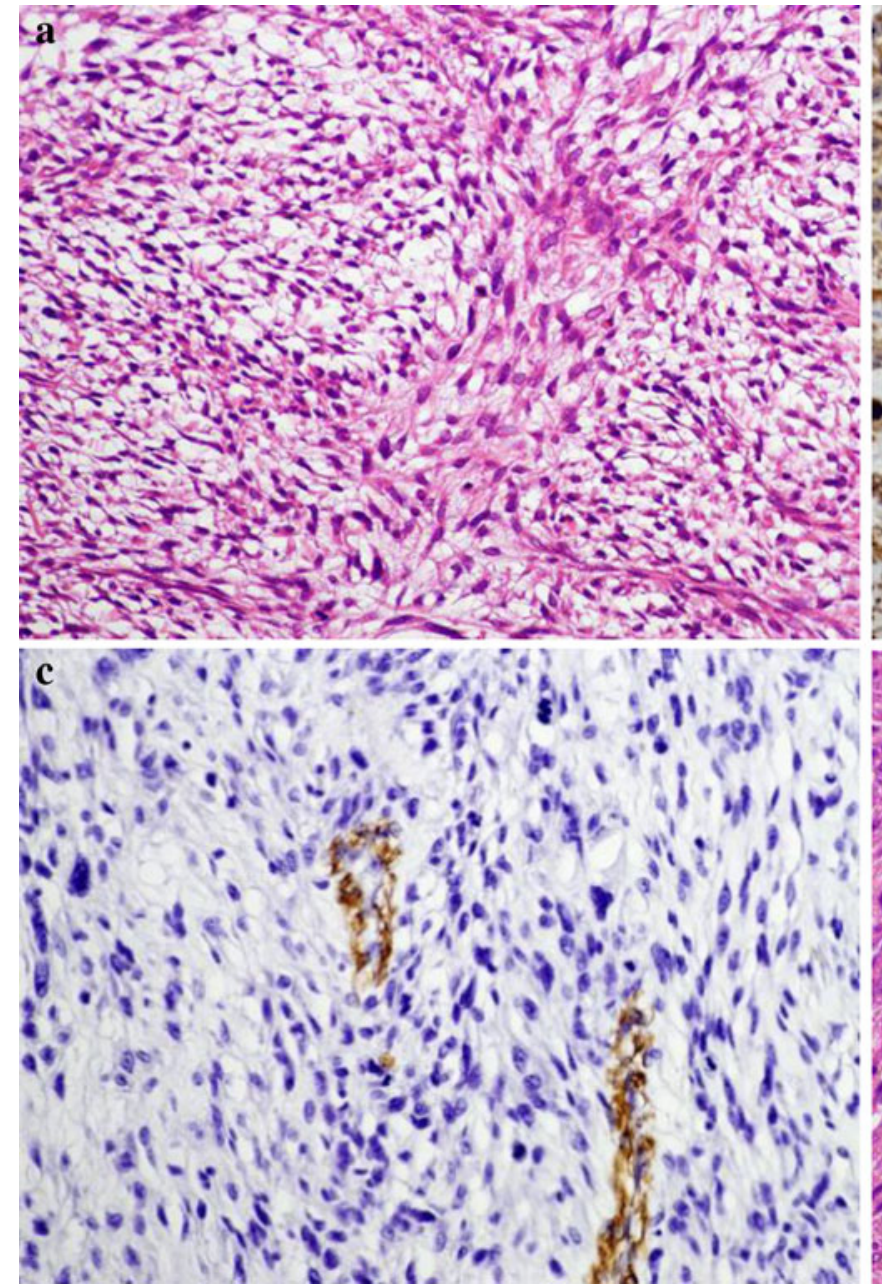

Fig. 2 Microscopic overview of the scrotal tumor showing myxofibrosarcoma. (H\&E stain $\times 50)($ a). Immunohistochemical studies were strongly positive for vimentin (b) and partially positive for

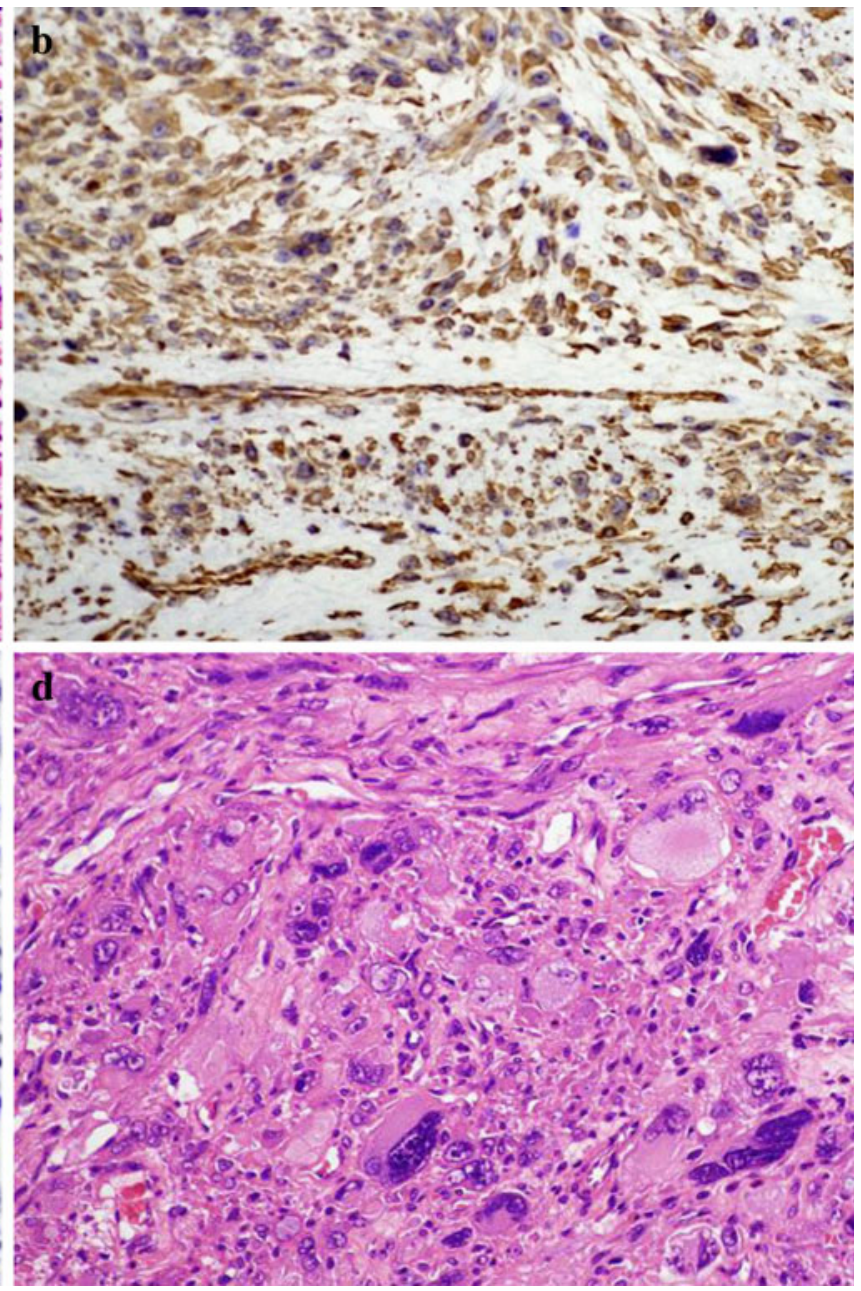

alpha-smooth muscle actin (c). Microscopic overview of the rectal tumor showing leiomyosarcoma $(\mathrm{H} \& \mathrm{E}$ stain $\times 50)(\mathbf{d})$ 
Fig. 3 CT scan before (a) and after (b) 4 courses of MAID
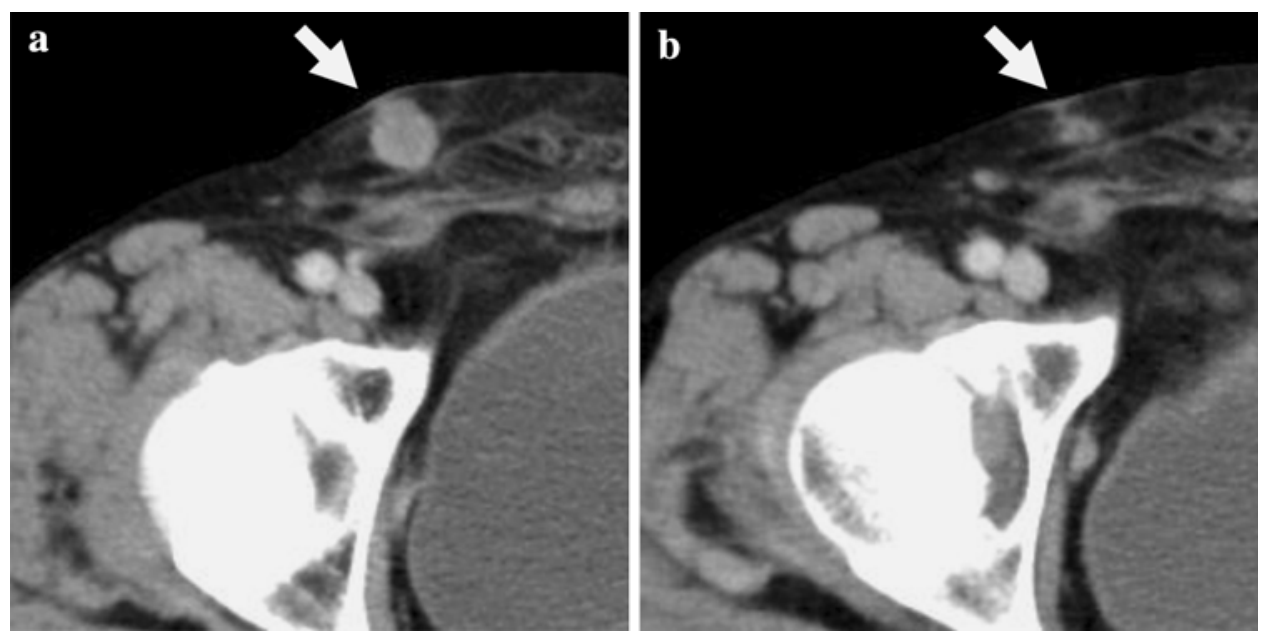

spindle cells with a pleomorphic storiform pattern were seen (Fig. 2a). Fusiform tumor cells varied from small and bland to enlarged, bizarre, pleomorphic, and multinucleated. Mitoses were frequent. Immunohistochemical studies were strongly positive for vimentin (Fig. 2b), partially positive for alpha-smooth muscle actin (Fig. 2c), and negative for desmin, muscle-specific actin (HHF35), and S100 in the tumor. The MIB-1 labeling index was 70\%. The scrotal tumor was histopathologically diagnosed as a T2bN0M0 MFS, high-grade, of the spermatic cord; the rectal tumor was diagnosed as a T1bN0M0 leiomyosarcoma (Fig. 2d).

Six months after the first operation, the patient underwent resection for a local recurrence and a left inguinal mass, which revealed a metastasis of MFS. But a CT scan 3 months after the second operation revealed local recurrences and lymph node metastasis of the left iliac area.

The patient was then rehospitalized for chemotherapy with a combination of mesna, doxorubicin, ifosfamide, and dacarbazine (MAID) therapy. A course of mesna $\left(2,500 \mathrm{mg} / \mathrm{m}^{2} /\right.$ day $)$ was administered on days $1-4$, and adriamycin (ADR) $\left(20 \mathrm{mg} / \mathrm{m}^{2} /\right.$ day), ifosfamide (IFOS) $\left(2,500 \mathrm{mg} / \mathrm{m}^{2} /\right.$ day $)$, and dacarbazine (DTIC) $\left(300 \mathrm{mg} / \mathrm{m}^{2} /\right.$ day) were administered daily on days $2-4$. The sizes of the two right inguinal recurrent tumors, left inguinal tumor, and left iliac lymph node before starting chemotherapy were $20.1,16.1,13.0$, and $20.7 \mathrm{~mm}$, respectively. A total of four courses of chemotherapy resulted in a $42 \%$ reduction in tumor size, which was judged as PR on the basis of the RECIST criteria (Fig. 3). Although leukopenia was doselimiting, there were no complications of fever. His 5th course required platelet transfusion for a platelet count $<20,000 / \mu 1$, although the chemical doses were reduced to $80 \%$ of the original. Nonhematologic toxicity was mild. Anorexia was controlled with antiemetics and vomiting was not seen. Cardiotoxicity was not detected. The patient has continued chemotherapy at a $50 \%$ dose and prolonged interval since his sixth course, maintaining PR for 8 months.

\section{Discussion}

Myxofibrosarcoma was first described by Angervall et al. [2] as a group of fibroblastic lesions with variability in the amount of myxoid stroma and representing a distinct clinicopathologic entity. Most of these tumors arise from the limbs, especially the lower extremities. They are rarely seen on the trunk. Tumors originating from the scrotum are extremely uncommon. Genitourinary sarcomas, making up $2.7 \%$ of all sarcomas, are uncommon in adults. Paratesticular sarcomas are even rarer, constituting less than $1 \%$ of all sarcomas [3]. Most patients present with a slowly enlarging and painless mass. As there are no characteristic radiological or biochemical findings indicating MFS, it is difficult to diagnose it before surgery. Histological examination, including immunohistochemical staining, is necessary for diagnosis. Although surgery is the most common form of therapy, local recurrence occurs in up to 50-60\% of cases. Lymph node metastatis is a rare event, and removal of regional lymph nodes is generally unnecessary, unless there is strong evidence of lymph node involvement. Takezawa et al. [4] reviewed cases of MFS of the spermatic cord in Japan, which showed sufficient local control was achieved by high orchiectomy.

There is a limited number of papers dealing with radiotherapy and chemotherapy for this disease, and neither therapy has been shown to be effective [5]. Because of the rarity of cases, no convincing data regarding survival times are yet available. Rational therapeutic regimens for metastatic soft-tissue sarcomas have not been established. ADR is the most effective single agent, with a response of 16-27\% for treatment of soft-tissue sarcomas [6-8]. Response for other standard single agents is less than $20 \%$. Large randomized studies, comparing ADR-based combination chemotherapy regimens with single-agent ADR, have been reported. In some of these trials response was greater in the combination chemotherapy arms [6-8] 
whereas in others primary outcomes were not significantly different between treatments. MAID, which we chose in this case, is a recommended combination regimen for softtissue sarcoma according to the National Comprehensive Cancer Network guidelines [9]. Elias et al. [10] have described the results of a phase II clinical trial of a combination of mesna, ADR, IFOS, and DTIC for patients with metastatic or unresectable sarcoma, and reported response of $47 \%$. The dose-limiting factor for MAID therapy is myelosuppression, as we experienced in the present case. Although the follow-up period is short, PR with no progression of the tumor has been observed for our patient. Careful long-term follow-up, however will be essential.

Conflict of interest The authors declare that we have no conflict of interest.

\section{References}

1. Mentzel T, Calonje E, Wadden C et al (1996) Myxofibrosarcoma. Clinicopathologic analysis of 75 cases with emphasis on the lowgrade variant. Am J Surg Pathol 20:391-405

2. Angervall L, Kindblom LG, Merck C (1977) Myxofibrosarcoma. A study of 30 cases. Acta Pathol Microbiol Scand A 85A:127-140
3. Russo P, Brady MS, Conlon K et al (1992) Adult urological sarcoma. J Urol 147:1032-1036

4. Takezawa K, Matsuoka Y, Takao T et al (2008) Myxofibrosarcoma of the spermatic cord: a case report. Jpn J Urol 99:555-559

5. Merck C, Angervall L, Kindblom LG, Odén A (1983) Myxofibrosarcoma: a malignant soft tissue tumor of fibroblastic-histiocytic origin. A clinicopathologic and prognostic study of 110 cases using multivariate analysis. Acta Pathol Microbiol Immunol Scand Suppl 282:1-40

6. Schoenfeld DA, Rosenbaum C, Horton J, Wolter JM, Falkson G, DeConti RC (1982) A comparison of adriamycin versus vincristine and adriamycin, and cyclophosphamide versus vincristine, actinomycin-D, and cyclophosphamide for advanced sarcoma. Cancer 50:2757-2762

7. Omura GA, Major FJ, Blessing JA et al (1983) A randomized study of adriamycin with and without dimethyl triazenoimidazole carboxamide in advanced uterine sarcomas. Cancer 52:626-632

8. Edmonson JH, Ryan LM, Blum RH et al (1993) Randomized comparison of doxorubicin alone versus ifosfamide plus doxorubicin or mitomycin, doxorubicin, and cisplatin against advanced soft tissue sarcomas. J Clin Oncol 11:1269-1275

9. Demetri GD, Antonia S, Benjamin RS, National Comprehensive Cancer Network Soft Tissue Sarcoma Panel et al (2010) Soft tissue sarcoma. J Natl Compr Canc Netw 8(6):630-674

10. Elias A, Ryan L, Sulkes A, Collins J, Aisner J, Antman KH (1989) Response to mesna, doxorubicin, ifosfamide, and dacarbazine in 108 patients with metastatic or unresectable sarcoma and no prior chemotherapy. J Clin Oncol 7:1208-1216 